\title{
A eficiência de um programa de reabilitação audiológica em idosos com presbiacusia e seus familiares
}

\section{The efficiency of an auditory rehabilitation program in elderly people with presbycusis and their family}

\author{
Christine Vieira Ruschel ${ }^{1}$, Claudia Ribeiro de Carvalho $^{2}$, Ana Cristina Guarinello ${ }^{3}$
}

\begin{abstract}
RESUMO
Objetivo: O objetivo dessa pesquisa foi desenvolver um programa de treinamento de estratégias de comunicação para indivíduos idosos, portadores de deficiência auditiva, usuários de aparelhos de amplificação sonora individual e seus familiares. Além disso, objetivamos verificar a contribuição da participação de familiares no processo de reabilitação audiológica desses sujeitos e promover melhor adaptação dos mesmos em relação ao uso do aparelho auditivo. Métodos: Fizeram parte da pesquisa 30 indivíduos com presbiacusia da cidade de Cascavel (PR). A amostra foi divida em dois grupos, o grupo 1 (grupo controle) foi constituído por 15 indivíduos que receberam uma única sessão de orientação e o grupo 2 (grupo experimental) constituiu-se de 15 idosos que participaram de um programa de reabilitação fonoaudiológica fracionado em seis sessões. Resultados: Foram encontradas diferenças estatisticamente significativas entre os grupos controle e experimental, quando comparadas as entrevistas dos pacientes e familiares, em aspectos como: manuseio da prótese auditiva, uso do telefone com a prótese auditiva e uso do diálogo em ambientes ruidosos. Conclusões: Neste estudo constatou-se que os idosos tiveram necessidade de acompanhamento fonoaudiológico após a adaptação do aparelho auditivo, pois sozinhos, muitas vezes, não conseguiam manusear a prótese auditiva e utilizá-la de maneira adequada.
\end{abstract}

DESCRITORES: Métodos de comunicação total; Auxiliares de audição; Saúde do idoso; Presbiacusia/reabilitação; Relações familiares; Reabilitação de deficientes auditivos

\section{INTRODUÇÃO}

O envelhecimento é um processo que se refere a uma série de mudanças associadas à passagem do tempo. Este processo pode trazer conseqüências ao indivíduo como: alterações biológicas, fisiológicas e psicológicas ${ }^{(1)}$.

Um estudo ${ }^{(1)}$ relata que a deficiência auditiva em idosos ou presbiacusia ocorre numa incidência de 5 a $20 \%$ em pes-

(1) Fonoaudióloga do Centro de Saúde do Trabalho - Porto Alegre (RS), Brasil; Especialista em Audiologia Clínica pela Universidade Tuiuti do Paraná - UTP - Curitiba (PR), Brasil.

(2) Fonoaudióloga do Centro Auditivo Cascavel - CAC - Cascavel (PR), Brasil; Especialista em Audiologia Clínica pela Universidade Tuiuti do Paraná - UTP - Curitiba (PR), Brasil.

(3) Doutora em Estudos Lingüísticos pela Universidade Federal do Paraná UFPR - Curitiba (PR), Brasil; Docente do Curso de Graduação em Fonoaudiologia, da Especialização em Audiologia Clínica e do Mestrado em Distúrbios da Comunicação da Universidade Tuiuti do Paraná - UTP - Curitiba (PR), Brasil.

Trabalho realizado na Universidade Tuiuti do Paraná- UTP - Curitiba (PR), Brasil.

Endereço para correspondência: Christine Vieira Ruschel. R. General Câmara, 395/101, Porto Alegre - RS, CEP 90010-230. E-mail: christineruschel@hotmail.com

Recebido em: 22/3/2007; Aceito em: 28/5/2007 soas de pelo menos 60 anos de idade e cerca de $60 \%$ em pessoas com mais de 60 anos de idade.

A presbiacusia tem como principal conseqüência um declínio na capacidade comunicativa do indivíduo, o que tende a isolá-lo e privá-lo das fontes de informação e comunicação, maximizando ainda mais as alterações causadas pelo envelhecimento ${ }^{(1)}$.

Com a perda da audição, em geral, surgem sentimentos de insegurança, medo e até a incapacidade, além disso, a dúvida quanto a possível progressão de perda é algo que pode deixar o adulto inquieto. As dificuldades de comunicação fazem com que o idoso duvide de suas capacidades e habilidades, tanto no âmbito profissional, quanto no pessoal, levando à mudanças na sua qualidade de vida, depressão e isolamento ${ }^{(2)}$.

Os problemas acometidos pela privação sensorial da audição podem ser minimizados com o uso do Aparelho de Amplificação Sonora Individual (AASI), o qual permite o resgate da percepção dos sons da fala e dos sons ambientais, promovendo a melhora da habilidade de comunicação.

Segundo a literatura ${ }^{(3)}$, a prevalência de uso de Aparelho de Amplificação Sonora Individual entre idosos é baixa, e o primeiro ano após a aquisição, é considerado o período crítico de adaptação, dentro do qual há risco de desistência. 
Atualmente o processo de escolha de um sistema de amplificação depende de um programa de reabilitação audiológica global que auxilie o portador de deficiência auditiva, bem como seus familiares, a lidarem com as desvantagens e incapacidades resultantes desta deficiência ${ }^{(4-5)}$.

Programas de reabilitação auditiva em idosos vem sendo alvo de pesquisas, sendo que diferentes estudos apontam resultados semelhantes sobre a eficácia desses programas ${ }^{(6-17)}$. Um exemplo é um estudo(5) ${ }^{(5)}$ sobe o programa de reabilitação audiológica para idosos, novos usuários de aparelho de amplificação sonora individual, avaliou por meio de um questionário, a autopercepção do handicap auditivo destes indivíduos quando comparados a um grupo não submetido ao programa de reabilitação. Participaram dessa pesquisa indivíduos com idade entre 63 e 88 anos, portadores de perda auditiva neurossensorial de grau moderado a severo, e novos usuários de AASI retroauricular. $\mathrm{O}$ estudo demonstrou que houve uma redução na autopercepção do handicap auditivo do grupo experimental quando comparado ao grupo controle.

Em um estudo ${ }^{(18)}$ sobre as dificuldades encontradas no processo de adaptação de aparelho de amplificação sonora individual em indivíduos idosos e a importância da participação da família neste processo, foram aplicados questionários aos idosos e a família. Com a análise dos questionários, concluiu-se que os idosos têm mais dificuldades nas atividades de usar o telefone, ouvir rádio e televisão, bem como manusear o próprio AASI, porém, segundo os idosos, a família foi imprescindível no processo de auxiliá-los a superar tais dificuldades.

Outros estudos ${ }^{(19-20)}$, concluem que os serviços de saúde da rede pública, que envolvem médicos e fonoaudiólogos, devem estabelecer diretrizes para o desenvolvimento de programas de diagnóstico, aquisição de aparelhos de amplificação sonora individual e, principalmente, de reeducação auditiva para os idosos portadores de presbiacusia, para que eles possam participar e desfrutar das relações sociais, mantendo uma boa qualidade de vida.

De acordo com os dados relatados acima, percebe-se que os programas de reabilitação auditiva para idosos são fundamentais para seu ajuste na família e na sociedade, desta forma, foi objetivo dessa pesquisa desenvolver um programa de treinamento de estratégias de comunicação para indivíduos idosos portadores de deficiência auditiva, usuários de AASI e seus familiares. Além disso, procurou-se verificar a contribuição da participação de familiares no processo de reabilitação audiológica dos sujeitos em questão e promover melhor adaptação dos sujeitos idosos em relação ao uso de AASI.

\section{MÉTODOS}

Essa pesquisa foi aprovada pelo Comitê de Ética em Pesquisa em Humanos e Animais da Universidade Tuiuti do Paraná, protocolo 027/2006. Antes da realização das orientações referentes ao programa de reabilitação, todos os participantes foram esclarecidos sobre o estudo, após o esclarecimento assinaram o Termo de Consentimento.

Fizeram parte dessa pesquisa 30 indivíduos com presbiacusia, da cidade de Cascavel com idade entre 60 e 87 anos e seus familiares (não definidos). Todos os idosos eram novos usuários de aparelho de amplificação sonora individual. A amostra foi divida em dois grupos, 15 indivíduos fizeram parte do grupo controle que recebeu uma única sessão de orientação e 15 do grupo experimental que participou de um programa de reabilitação fonoaudiológica fracionado em seis sessões. Na primeira sessão, todos os idosos e familiares receberam orientação sobre as instruções necessárias para conseguir fazer uso do AASI durante a semana. Da segunda sessão em diante apenas o grupo experimental e seus familiares receberam orientações. Cabe ressaltar que nem todos os familiares que participaram da pesquisa moravam com os idosos.

$\mathrm{Na}$ primeira sessão foram realizadas orientações com relação ao uso da prótese auditiva para os dois grupos de pacientes e seus familiares. Ainda na primeira sessão foram aplicados os questionários para os pacientes (Anexo 1) e familiares (Anexo 2) dos dois grupos. Nas cinco sessões restantes, apenas o grupo experimental e seus familiares receberam orientações quanto às estratégias de comunicação. Além disso, durante essas sessões de orientação, os pacientes e familiares puderam esclarecer dúvidas e falar sobre a adaptação da prótese auditiva. Na última sessão foram aplicados novamente os mesmos questionários para os pacientes e familiares do grupo experimental.

\section{RESULTADOS}

Os dados descritos abaixo foram analisados através da estatística simples e dos testes de Fisher e Qui-quadrado. Cabe ressaltar que somente as perguntas que apresentaram diferenças nas respostas foram analisadas, portanto as questões $\mathrm{n}^{\mathrm{o}} 1,2,3,4,5,7,8,9,10,13$ e 14 , realizadas com os pacientes (Anexo 1) e questões $n^{\circ} 1,2,3,5$ e 7 , realizadas com os familiares (Anexo 2) não foram analisadas.

Quando comparados o grupo controle de pacientes com a segunda entrevista do grupo experimental, obtivemos as seguintes respostas:

Na Figura 1 observam-se as respostas dos pacientes quanto às dificuldades para retirar e colocar a prótese auditiva do ouvido.

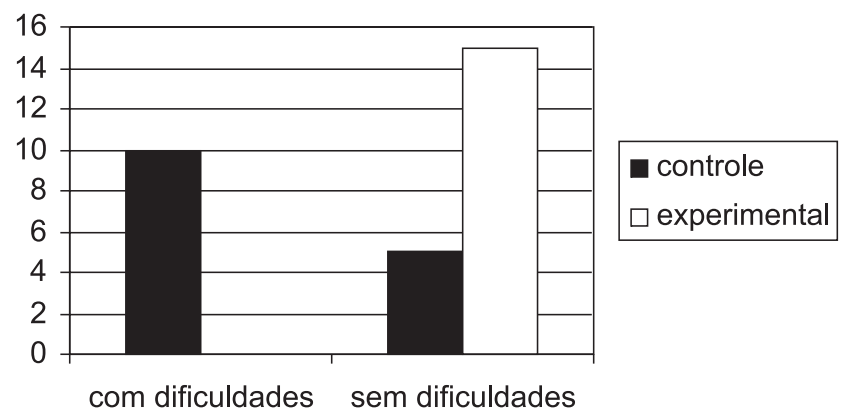

Figura 1. Freqüência das dificuldades do grupo controle e do grupo experimental para colocar e retirar o AASI

Percebe-se nessa figura que mais da metade dos idosos do grupo controle referiram na primeira sessão que tinham dificuldades para colocar e retirar o aparelho auditivo, já no 
grupo experimental, após as cinco sessões de orientação, notase que nenhum dos idosos apresentou dificuldades. Nesta pergunta foi aplicado o teste de Fisher, por meio do qual verificou-se que houve uma diferença significativa entre os resultados dos dois grupos, $\mathrm{p}=0,0001<0,05$. Os pacientes do grupo controle que tiveram apenas uma sessão de orientação continuaram com dúvidas com relação a colocação do aparelho auditivo, o que não aconteceu com o grupo experimental, que passou por seis sessões, e pode aprender corretamente como se coloca e retira a prótese auditiva.

Na Figura 2 observam-se as respostas dos participantes com relação ao incômodo da prótese auditiva em ambientes ruidosos.

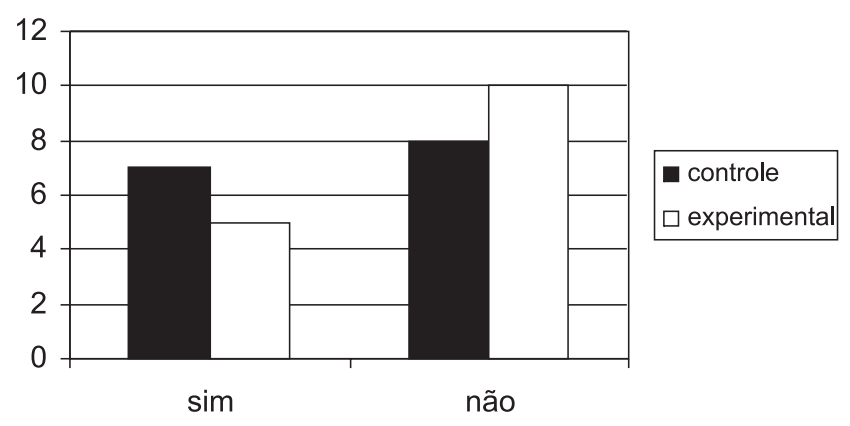

Figura 2. Freqüência de incômodo em ambientes ruidosos

Para esta questão foi aplicado o teste de Qui-quadrado, e não houve diferença significativa entre os dois grupos, pois em ambos a maioria dos pacientes referiu não sentir desconforto com relação aos ambientes ruidosos.

Na Figura 3 abaixo foram comparadas a primeira e a segunda entrevistas do grupo experimental com relação às dificuldades para retirar e colocar a prótese auditiva do ouvido. Percebe-se nesta figura que na primeira entrevista todos os idosos responderam que não sabiam retirar e colocar a prótese auditiva no ouvido e, na segunda entrevista, todos responderam que sim, ou seja, que sabiam retirar e colocar a prótese auditiva no ouvido.

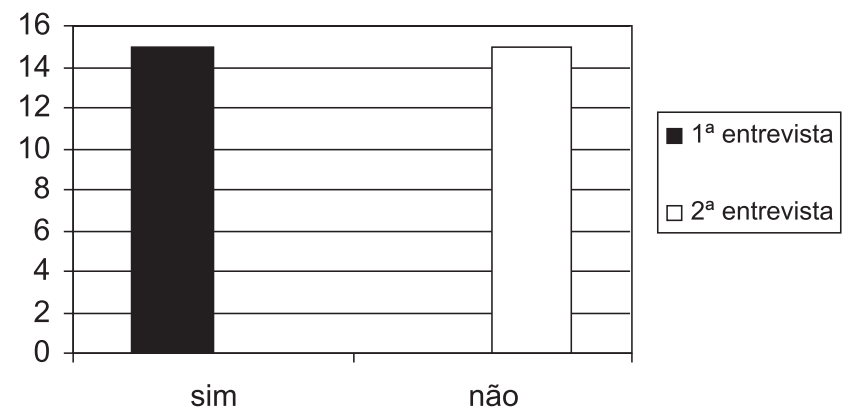

Figura 3 - Freqüência de dificuldades para colocar e retirar o AASI no grupo experimental

Na Figura 4 observam-se as respostas dos participantes do grupo experimental com relação ao incômodo com a prótese auditiva em ambientes ruidosos.

Na Figura 4 foi aplicado o teste de Qui-quadrado, e no-

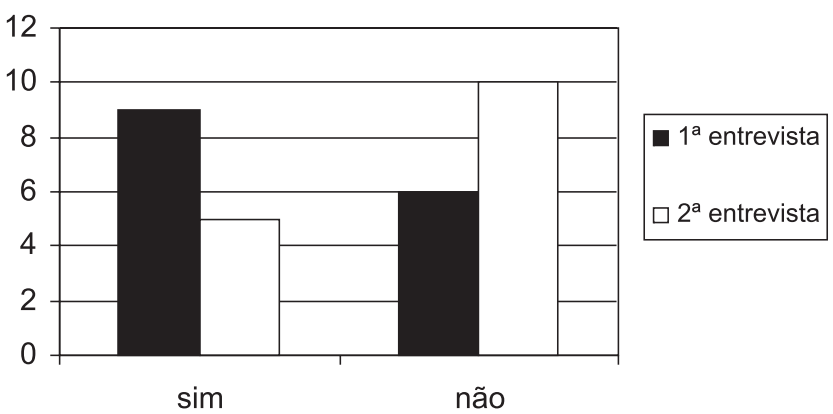

Figura 4. Freqüência de incômodo do grupo experimental em ambientes ruidosos

vamente não houve diferença significativa entre a primeira e a última sessão, porém pode-se perceber que após as sessões de orientação diminuíram os incômodos com relação ao uso da prótese auditiva em ambientes ruidosos.

Na Figura 5 observam-se as respostas dos pacientes em relação à dificuldade ao falar no telefone com a prótese auditiva.

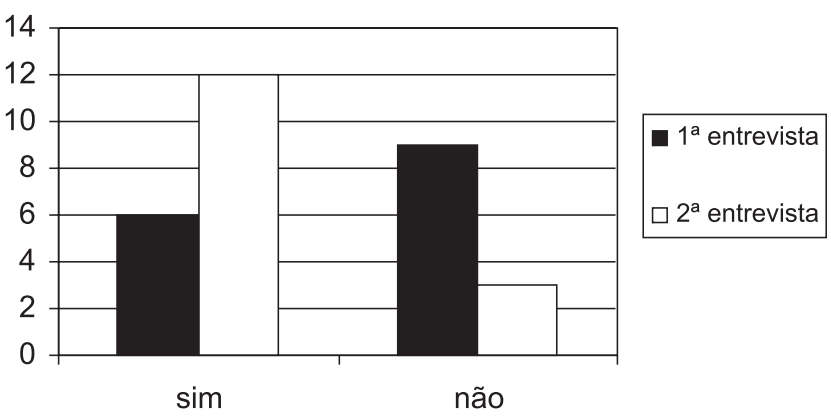

Figura 5. Freqüência de dificuldade ao falar ao telefone no grupo experimental

Percebe-se na Figura 5 que inicialmente a maioria dos pacientes tinha dificuldades para falar ao telefone e após as sessões essa dificuldade foi sanada. Nesta pergunta o teste de Fisher foi aplicado, tendo como resultado um $\mathrm{p}=0,0302$, ou seja, para um nível de significância de 5\% houve diferença significativa nos resultados da primeira entrevista e da segunda entrevista do grupo experimental.

Com relação às análises das respostas dos familiares do grupo controle e do grupo experimental quando comparadas a primeira e a segunda entrevistas, as Tabelas 1, 2, 3, 4, 5 e 6 mostram que houve uma diferença significativa entre os dois grupos.

A Tabela 1 mostra as respostas dos familiares com relação ao manuseio da prótese auditiva do paciente.

Observa-se na Tabela 1, que no grupo controle e no grupo experimental a maioria dos familiares durante a primeira entrevista respondeu que seus familiares não sabiam manejar a prótese auditiva. Após a segunda entrevista percebe-se que os pacientes do grupo experimental aprenderam a manejar a prótese auditiva.

A Tabela 2 mostra as respostas dos familiares quanto ao auxilio sobre o manuseio da prótese auditiva.

Observa-se na Tabela 2, que após as sessões de reabilitação auditiva, o número de familiares capazes de ajudar os 
Tabela 1. Percepção do familiar com relação ao manejo da prótese auditiva pelo idoso

\begin{tabular}{|c|c|c|c|c|c|c|}
\hline & \multicolumn{2}{|c|}{ G. Controle } & \multicolumn{2}{|c|}{$1^{\mathrm{a}}$ Entrev. G. Experimental } & \multicolumn{2}{|c|}{$2^{a}$ Entrev. G. Experimental } \\
\hline & (n) & $\%$ & (n) & $\%$ & (n) & $\%$ \\
\hline Sabe manejar & 5 & 33 & 4 & 27 & 14 & 93 \\
\hline Total & 15 & 100 & 15 & 100 & 15 & 100 \\
\hline
\end{tabular}

Tabela 2. Ajuda do familiar com relação ao manejo da prótese auditiva

\begin{tabular}{|c|c|c|c|c|c|c|}
\hline & \multicolumn{2}{|c|}{ G. Controle } & \multicolumn{2}{|c|}{$1^{a}$ Entrev. G. Experimental } & \multicolumn{2}{|c|}{$2^{\mathrm{a}}$ Entrev. G. Experimenta } \\
\hline & $(n)$ & $\%$ & (n) & $\%$ & (n) & $\%$ \\
\hline Familiar ajuda & 9 & 60 & 10 & 67 & 14 & 93 \\
\hline Total & 15 & 100 & 15 & 100 & 15 & 100 \\
\hline
\end{tabular}

idosos a manusear a prótese auditiva aumentou.

A Tabela 3 mostra as respostas da percepção dos familiares com relação ao uso do telefone pelo idoso.

Observa-se na Tabela 3 que após a adaptação do aparelho auditivo, os participantes do grupo experimental estão conseguindo ouvir bem ao telefone.

A Tabela 4 mostra as respostas dos familiares com relação ao prosseguimento do diálogo dos pacientes em ambientes ruidosos.

Observa-se na Tabela 4 que, após as seis sessões de terapia, os pacientes do grupo experimental estão conseguindo prosseguir com o diálogo em ambientes ruidosos.
A Tabela 5 mostra as respostas dos familiares quanto à situação do paciente pedir para repetir, caso não entenda o que foi dito.

Observa-se na Tabela 5 que, ao final das seis sessões, os pacientes do grupo experimental não têm necessidade de que outras pessoas repitam novamente o que estavam falando.

Por fim, a Tabela 6 mostra as respostas dos familiares quanto à comunicação do paciente após a colocação da prótese auditiva.

Observa-se na Tabela 6 que os pacientes de ambos os grupos estão mais comunicativos, porém a porcentagem é maior no grupo experimental.

Tabela 3. Percepção do familiar com relação ao uso do telefone pelo idoso

\begin{tabular}{|c|c|c|c|c|c|c|}
\hline & \multicolumn{2}{|c|}{ G. Controle } & \multicolumn{2}{|c|}{$1^{a}$ Entrev. G. Experimental } & \multicolumn{2}{|c|}{$2^{\mathrm{a}}$ Entrev. G. Experimenta } \\
\hline & $(\mathrm{n})$ & $\%$ & (n) & $\%$ & (n) & $\%$ \\
\hline Usa bem o telefone & 9 & 60 & 4 & 27 & 14 & 93 \\
\hline Não usa bem o telefone & 1 & 7 & 5 & 33 & 0 & 0 \\
\hline Não fez o teste & 5 & 33 & 4 & 27 & 0 & 0 \\
\hline Não sabe informar & 0 & 0 & 2 & 13 & 1 & 7 \\
\hline Total & 15 & 100 & 15 & 100 & 15 & 100 \\
\hline
\end{tabular}

Tabela 4. Percepção do familiar quanto ao diálogo do idoso em ambientes ruidosos

\begin{tabular}{|c|c|c|c|c|c|c|}
\hline & \multicolumn{2}{|c|}{ G. Controle } & \multicolumn{2}{|c|}{$1^{\text {a }}$ Entrev. G. Experimental } & \multicolumn{2}{|c|}{$2^{a}$ Entrev. G. Experimental } \\
\hline & (n) & $\%$ & (n) & $\%$ & (n) & $\%$ \\
\hline Mantém o diálogo & 9 & 60 & 10 & 66 & 13 & 86 \\
\hline Não mantém o diálogo & 2 & 13 & 3 & 20 & 1 & 7 \\
\hline Às vezes mantém o diálogo & 0 & 0 & 1 & 7 & 0 & 7 \\
\hline Não sabe informar & 4 & 27 & 1 & 7 & 1 & 0 \\
\hline Total & 15 & 100 & 15 & 100 & 15 & 100 \\
\hline
\end{tabular}

Tabela 5. Percepção do familiar com relação ao idoso pedir para que repitam o que lhe dizem

\begin{tabular}{|c|c|c|c|c|c|c|}
\hline & \multicolumn{2}{|c|}{ G. Controle } & \multicolumn{2}{|c|}{$1^{a}$ Entrev. G. Experimental } & \multicolumn{2}{|c|}{$2^{a}$ Entrev. G. Experimental } \\
\hline & (n) & $\%$ & (n) & $\%$ & (n) & $\%$ \\
\hline Pede que repitam & 2 & 13 & 2 & 13 & 0 & 0 \\
\hline Não pede que repitam & 2 & 13 & 6 & 40 & 0 & 0 \\
\hline Às vezes pede que repita & 4 & 47 & 1 & 7 & 0 & 0 \\
\hline Não há necessidade de repetir & 7 & 27 & 6 & 40 & 15 & 100 \\
\hline Total & 15 & 100 & 15 & 100 & 15 & 100 \\
\hline
\end{tabular}


Tabela 6. Percepção do familiar com relação a comunicação do idoso após a adaptação da prótese auditiva

\begin{tabular}{|c|c|c|c|c|c|c|}
\hline & \multicolumn{2}{|c|}{ G. Controle } & \multicolumn{2}{|c|}{$1^{\text {a }}$ Entrev. G. Experimental } & \multicolumn{2}{|c|}{$2^{a}$ Entrev. G. Experimental } \\
\hline & (n) & $\%$ & (n) & $\%$ & (n) & $\%$ \\
\hline Mais comunicativo & 9 & 60 & 12 & 80 & 15 & 100 \\
\hline Menos comunicativo & 1 & 7 & 0 & 0 & 0 & 0 \\
\hline A mesma coisa & 5 & 33 & 3 & 20 & 0 & 0 \\
\hline Total & 15 & 100 & 15 & 100 & 15 & 100 \\
\hline
\end{tabular}

\section{DISCUSSÃO}

Como é possível visualizar nas Figuras 1 e 3, houve diferença significativa da primeira para a segunda entrevista do grupo experimental quando comparados com o grupo controle com relação ao manuseio da prótese auditiva. Percebeu-se que o grupo experimental a partir da segunda sessão de orientação apresentou dúvidas quanto à higienização do molde, a colocação do aparelho auditivo, a recolocação do molde após a higienização, a dificuldades para trocar as pilhas etc. Estes achados vêm ao encontro com o que foi encontrado em alguns estudos ${ }^{(18)}$. As autoras afirmam que no início da adaptação auditiva os idosos tiveram várias dificuldades com relação ao manuseio da prótese auditiva.

Após as seis sessões de terapia, os pacientes relataram que não apresentavam mais nenhuma dificuldade com relação a higienização, manuseio e lado da prótese auditiva. Referiram também que voltaram a conversar pelo telefone com os amigos e que foi muito boa a sensação de não ter que pedir para os outros falarem mais alto durante a conversa. Todos disseram que após as sessões de orientação estão mais comunicativos, pois antes ficavam inseguros durante as conversas, já que tinham dificuldades para se ouvir e ouvir aos outros. Esses resultados estão de acordo com alguns estudos citados anteriormente na literatura ${ }^{(3,9-10,12-14)}$.

Notou-se também a importância do acompanhamento familiar, uma vez que, em geral os idosos que fazem adaptação da prótese auditiva sem acompanhamento costumam retornar por não conseguir colocar o aparelho ou manusear o controle de volume, acarretando, desta forma, o não uso do aparelho auditivo ${ }^{(18)}$. Já os idosos acompanhados por membros da família declararam que houve dificuldades, mas que o familiar que o acompanhava estava ajudando na superação das dificuldades, dentre elas, o manuseio da prótese auditiva e os diálogos para melhorar a comunicação.

Os familiares relataram que após as seis sessões de terapia, estavam felizes por seus parentes estarem mais comunicativos e alegres por ouvirem os sons que antes pareciam impossíveis de se escutar. Referiram também, que ficaram muito gratificados, pois afinal eles contribuíram para que esse processo de adaptação da prótese auditiva desse certo.

O programa de acompanhamento fonoaudiológico e a ajuda da família foram fatores essenciais no processo de reabilitação auditiva dos idosos entrevistados nesta pesquisa. Portanto, como segundo a Organização Mundial de Saúde (OMS), até 2025 o Brasil será o $6^{\circ}$ país do mundo com o maior número de pessoas idosas, as pesquisas futuras nessa área são extremamente relevantes. Cabe aos fonoaudiólogos e todas as pessoas relacionadas à saúde propor programas de reabilitação auditiva e esclarecer a população sobre seus benefícios, já que de nada adianta apenas comprar ou receber os aparelhos auditivos e não conseguir utilizá-los devido à falta de adaptação e orientação.

\section{CONCLUSÃO}

Neste estudo constatou-se que os idosos têm necessidade de acompanhamento fonoaudiológico após a adaptação do aparelho auditivo, pois sozinhos, muitas das vezes, não conseguem fazer todo o processo de higienização e manuseio da prótese auditiva. Pode-se constatar também, que os pacientes melhoraram sua performance comunicativa e os diálogos em ambientes ruidosos e, recomeçaram a utilizar o telefone diariamente.

Da mesma forma, pode-se ratificar a importância dos familiares na recolocação deste idoso junto ao convívio social da família, dos amigos e dos companheiros de trabalho.

Conclui-se, portanto, que o trabalho audiológico nesta faixa etária da população é muito complexo, e requer do fonoaudiólogo a sensibilidade e empatia na compreensão das dificuldades enfrentadas pelo paciente. Além disso, esse profissional deve possuir conhecimentos sobre o envelhecimento e suas consequiências para então, obter melhores resultados na reabilitação auditiva. É preciso ainda, que dentro deste contexto sejam inseridos os familiares mais próximos, os companheiros de trabalho e a sociedade como um todo, por meio de campanhas de alcance nacional, para que se possa vencer o preconceito, ainda existente, e recolocar o idoso no convívio social. 


\begin{abstract}
Purpose: The aim of this research consisted on developing a training program of communicative strategies for elderly people with auditory problems, users of individual hearing aids, and their families. Additionally, we had the aim to verify the contribution of family intervention during the process of auditory rehabilitation and to promote better adaptation of these individuals regarding the use of their hearing aids. Methods: Thirty elderly subjects with presbycusis from the city of Cascavel (PR - Brazil) took part in this research. The sample was divided into two groups: group 1 (control group) comprised 15 individuals who received only one orientation session; group 2 (experimental group) comprised another 15 individuals who took part in an audiological rehabilitation program of six sessions. Results: Statistically significant differences were found between control and experimental groups when patients' and their families' interviews were compared, especially regarding: handling the hearing aid itself, using the telephone with the hearing aid and performing dialogues in noisy environments. Conclusions: The study showed that the elderly people investigated needed orientation follow-up sessions after the hearing aid adjustment, because they had difficulties handling and using the prosthesis in a proper way.
\end{abstract}

Keywords: Communication methods, total; Hearing aids; Health of the elderly; Presbycusis/rehabilitation; Family relations; Rehabilitation of hearing impaired

\title{
REFERÊNCIAS
}

1. Kieling CH. Reabilitação audiológica em idosos [monografia]. Porto Alegre: CEFAC; 1999. [Apresentada como Trabalho Conclusão de Curso Especialização].

2. Souto DPV. A importância do fonoaudiólogo na adaptação da prótese auditiva [texto na Internet]. [citado 2005 Jun 19]. Disponível em: http://www.pedagobrasil.com.br/fonoaudiologia/ aimportanciadofonoaudiologo.htm

3. Tschiedel RS. Programa de reabilitação audiológica para idosos usuários de aparelhos de amplificação sonora individual e seus interlocutores mais freqüentes [tese]. Brasília: Universidade de Brasília. Instituto de Psicologia; 2003.

4. Almeida K, Iorio MCM. Próteses auditivas: fundamentos teóricos e aplicações clínicas. 2a ed. São Paulo: Lovise; c2003.

5. Boéchat EM. Ouvir sob o prisma da estratégia [tese]. São Paulo: Pontifícia Universidade Católica de São Paulo; 1992.

6. Souza AEN, Russo ICP. Um programa de reabilitação audiológica para idosos, novos usuários de aparelho de amplificação sonora individual. Pró-Fono. 1998;10(2):16-22.

7. Silva AS, Venites JP, Bilton TL. A relação entre o uso de aparelho de amplificação sonora individual - AASI - e a melhora da função cognitiva no envelhecimento. Distúrb Comun. 2002;14(1):63-89.

8. Marques ACO, Kozlowski L, Marques JM. Reabilitação auditiva no idoso. Rev Bras Otorrinolaringol. 2004;70(6):806-11.

9. Veiga LR, Merlo ARC, Mengue SS. Satisfação com a prótese auditiva na vida diária em usuários do Sistema de Saúde do Exército. Rev Bras Otorrinolaringol. 2005;71(1):67-73.

10. Guarinello AC, Cruz MCM. O perfil dos idosos protetizados na Clínica de Fonoaudiologia da Universidade Tuiuti do Paraná. Fono Atual. 2006;8(35):59-64.

11. Ferreira MIDC, Signor RC. O perfil do idoso usuário de prótese auditiva: um estudo da satisfação. Rev Fonoaudiol Bras. 2006;4(2):12.
12. Batista ACM, Sampaio FM. Nível de satisfação dos idosos usuários de próteses auditivas doadas pela APAC-NAMI-UNIFOR. RBPS: Rev Bras Prom Saúde. 2005;18(1):7-10.

13. Oliveira JRM, Motti TFG, Neuber DRD, Prado EP, Creppe SVTZ, Andrade CF. Dificuldades encontradas pelos deficientes auditivos adultos, após a adaptação do aparelho de amplificação sonora individual (AASI). Acta AWHO. 2001;20(4):201-5.

14. Jóia AC, Fioravanti MP, Tamashiro IA, Martins RHG, Montovani JC. Roteiro de acompanhamento e orientação fonoaudiológica em pacientes adultos deficientes auditivos após a adaptação de aparelho de amplificação sonora individual. Pró-Fono. 1997;9(1):62-5.

15. Pinzan-Faria VM, Iório MCM. Sensibilidade auditiva e autopercepção do handicap: um estudo em idosos. Distúrb Comun. 2004;16(3):28999.

16. Silman S, Iório MCM, Mizhahi MM, Parra VM. Próteses auditivas: um estudo sobre seu benefício na qualidade de vida de indivíduos portadores de perda auditiva neurossensorial. Distúrb Comun. 2004;16(2):153-65.

17. Bucuvic EC, Iório MCM. Benefício e dificuldades auditivas: um estudo em novos usuários de prótese auditiva após dois e seis meses de uso. Fono Atual. 2004;7(29):19-29.

18. Barros PFS, Queiroga BAM. As dificuldades encontradas no processo de adaptação de aparelho de amplificação sonora individual em indivíduos idosos. Rev CEFAC. 2006;8(3):375-85.

19. Veras RP, Mattos LC. Audiologia do envelhecimento: revisão da literatura e perspectivas atuais. Rev Bras Otorrinolaringol. 2007;73(1):128-34.

20. Iório MCM, Almeida K. Próteses auditivas: histórico e tendências atuais. Acta AWHO. 1990;9(2):44-8, 50-1. 
Anexo 1. Questionário aos pacientes

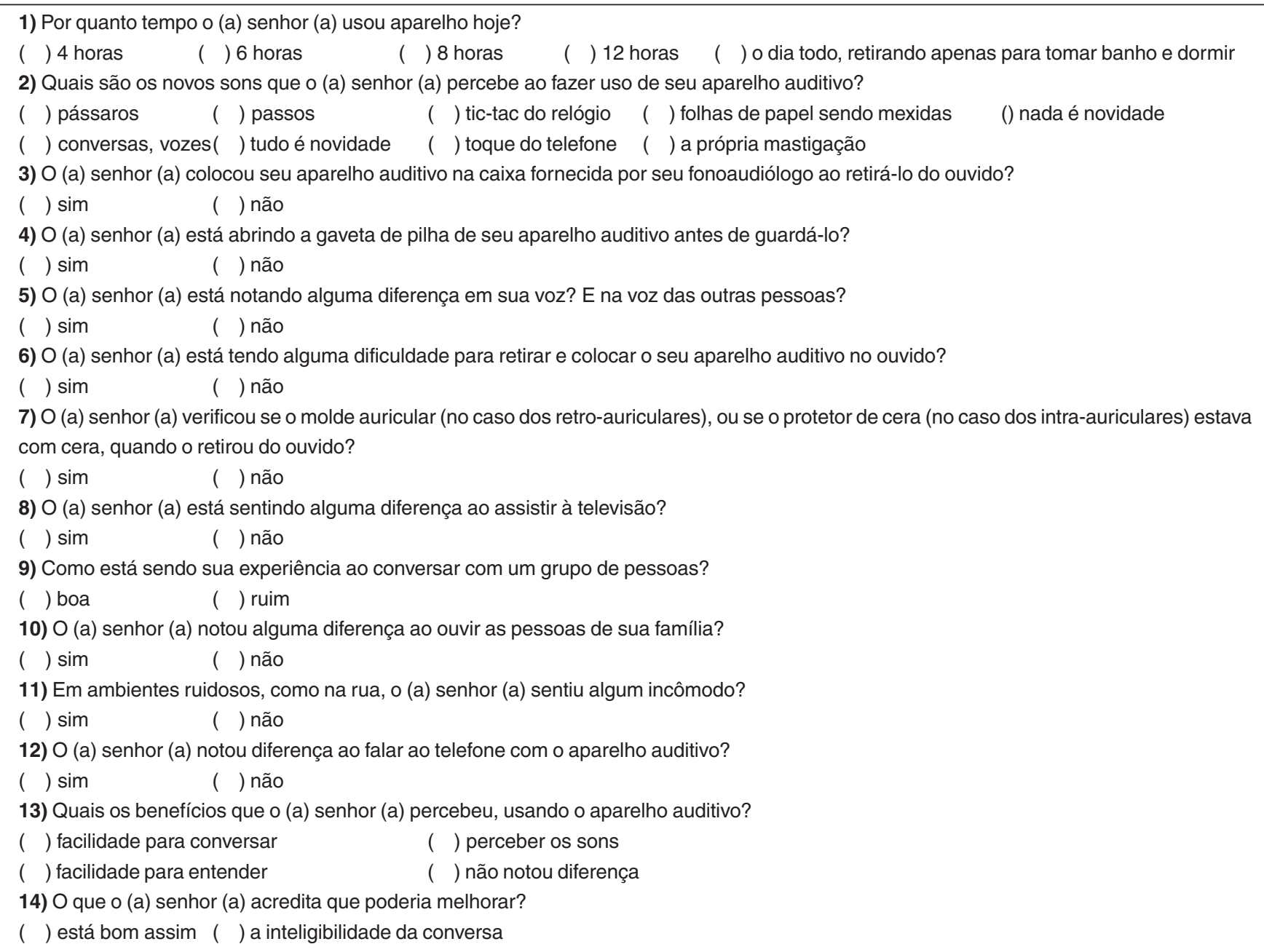

Anexo 2. Questionário aos familiares

1) O seu familiar está se adaptando com a prótese auditiva?

( ) $\operatorname{sim}($ ) não

2) Você está incluindo o seu familiar nas conversas do dia-a-dia?

( ) $\operatorname{sim}($ ) não

3) Você está tendo paciência ao falar com o seu familiar, de modo que ele o entenda?

( ) $\operatorname{sim}($ ) não

4) Seu familiar está sabendo manejar a prótese auditiva? E você, está sabendo como ajudá-lo?
( ) sim
( ) não
( ) mais ou menos
( ) não sabe informar
() $\operatorname{sim}$
( ) não

5) Quando estão assistindo televisão, ou ouvindo rádio, seu familiar ainda aumenta o volume consideravelmente dos mesmos?

( ) $\operatorname{sim}($ ) não

6) Você pode afirmar que o seu familiar está ouvindo bem ao telefone com a prótese auditiva?
( ) sim
( ) não
( ) ainda não fez o teste
( ) não sabe informar

7) Em ambientes ruidosos, como na rua, seu familiar não estranhou ouvir novamente os barulhos de carro, ônibus, buzinas, etc.?
() $\operatorname{sim}$
( ) não
( ) às vezes
( ) não sabe informar

8) Em ambientes com muito ruído, você acha que o seu familiar está conseguindo prosseguir com o diálogo? Ele não está se sentindo mal com a situação de pedir para as pessoas repetir novamente, caso não entenda o que falam?
( ) $\operatorname{sim}$
( ) não
( ) às vezes
( ) não sabe informar
( ) sim
( ) não
( ) ás vezes
( ) não há necessidade de repetir

9) Em relação à comunicação do seu familiar, como ela está após a colocação e adaptação da prótese auditiva?
( ) mais comunicativa
( ) menos comunicativa
( ) a mesma coisa 